\title{
On the Numerical Solutions of One and Two-Stage Model of Carcinogenesis Mutations with Time Delay and Diffusion*
}

\author{
Ishtiaq Ali \\ Department of Mathematics, COMSATS Institute of Information Technology, Islamabad, Pakistan \\ Email: ishtiaqali@comsats.edu.pk
}

Received August 15, 2013; revised September 15, 2013; accepted September 23, 2013

Copyright (C) 2013 Ishtiaq Ali. This is an open access article distributed under the Creative Commons Attribution License, which permits unrestricted use, distribution, and reproduction in any medium, provided the original work is properly cited.

\begin{abstract}
In this paper, we focused on numerical solutions of carcinogenesis mutations models that are based on reaction-diffusion systems and Lotka-Volterra food chains. We consider the case with one and two-stages of mutations with appropriate initial conditions and the zero-flux boundary conditions. The main purpose is to construct a stable discretization scheme, which allows much accuracy than those of a standard approach. To this end, we use the spectral method to postprocess numerical solutions for the proposed model by using some classical methods for solving differential equations. The implementation of the algorithm is simple and it does not need to solve the linear or nonlinear system (in case the model is nonlinear). We simulate the one and two-stage carcinogenesis mutations model and compared the results with previously published ones.
\end{abstract}

Keywords: Carcinogenesis Mutations; Delay and Diffusion; Spectral Postprocessing; Numerical Simulations

\section{Introduction}

The struggle for finding an effective and permanent cure for tumor continues to challenge scientists has been made by a lot of progresses in discovering new methodologies which are helpful in successful treatments to reduce and even clear tumors. Mathematical modeling is one of the tools to improve the cancer therapy. Carcinogenesis is a very complicated process and one need a comprehensive study to fully understand it. Tumors are derived from one or more normal cells that have undergone malignant transformation. The immune response to tumors depends on how antigenic the tumor is. A cell that has undergone significant mutation results in a tumor is easier to be recognized as foreign (i.e. more antigenic) than one that differs only slightly from a healthy cell [1]. For different types of cancers, it is possible to divide the process into different number of stages, normally between 4 and 7 stages, which depends on the type of tumor [2].

In this paper, we study a simple model of carcinogenesis mutations of DNA, which originally comes from [3]

\footnotetext{
*Numerical solutions of one and two-stage model.

This work was carried out during the tenure of an ERCIM "Alain Bensoussan" Fellowship Programme. This Programme is supported by the Marie Curie Co-funding of Regional, National and International Programmes (COFUND) of the "European Commission".
}

and was also studied in [4-9], which describe a process of carcinogenesis mutations with $n$ different steps of mutations (from normal to malignant cells). The model is expressed in terms of system of partial differential equation, in which the latest stage of mutation has different forms depending on whether it has growth advantage in favorable or competitive conditions or disadvantage of growth in unfavorable and competitive conditions. For simplicity in this paper, we only consider the latest stage in unfavorable conditions, as in the case of favorable conditions there is no possibility to cure the disease without any treatment. For more details we refer to $[10,11]$.

The current work provides the computational and implementational details needed to study the dynamics of these equations [12]. A detailed analysis of the one-stage model equations was undertaken in [4]. We use spectral methods to postprocess numerical solutions, which use the numerical solutions of a lower order method to serve as starting value of the spectral methods. The iteration uses the Gauss-Seidel type strategy, which can be very useful in terms of improving the accuracy of the numerical solutions. In particular for the problems in which accuracy is the only issue and some conservatives properties are even more important for large time simulation. Also there is no need to solve a linear or nonlinear sys- 
tem of equations as we do need in case of using some other numerical methods [13].

The paper is organized as follows. Section 2 and 3 is used for the description of models in detail. In Section 4 and 5 we describe in detail the spectral postprocessing approximation for the proposed models. Section 6 is used for the numerical simulations and discussions, followed by the concluding remarks in Section 7 .

\section{Formulation of the Model}

Let us consider $Y_{j}$ as a density of mutant cells of the $j$-th stage at position $(t, x)$ where $j=0,1,2,3, \cdots, n$. To develop a multistage model for mutant cells densities at stage $Y_{j}, j=0,1,2,3, \cdots, n$, where $Y_{0}$ will be the initial stage, $Y_{j}(j=1,2,3, \cdots, n-1)$ the density of intermediate stages, and $Y_{n}$ represents the density of the final stage. The system of equations for the density function is given by [2].

$$
\frac{\partial Y_{j}}{\partial t}=D_{j} \Delta Y_{j}+a_{i} Y_{j}\left(1-\frac{Y_{j}}{K_{j}}\right)+\eta_{j} Y_{j} Y_{j-1}-\mu_{j+1} Y_{j} Y_{j+1} .
$$

The final stage of mutation occurs when cancer cells become malignant and metastasize. We assume that for the malignant mutation in the final stage, the carrying capacity is unlimited; that is $K_{n} \rightarrow \infty$, thus Equation (1) becomes

$$
\frac{\partial Y_{n}}{\partial t}=D_{n} \Delta Y_{n}+a_{n} Y_{n}+\eta_{n} Y_{n} Y_{n-1}
$$

\section{Formulation of One and Two-Stage Mutation}

The full blown developed malignant mutation can be described in the following system of the $n$-stage model, that is initial, benign, and malignant,

$$
\begin{aligned}
& \frac{\partial Y_{0}}{\partial t}=D_{0} \Delta Y_{0}+a_{0} Y_{0}\left(1-\frac{Y_{0}}{K_{0}}\right)+\mu_{1} Y_{0} Y_{1}, \\
& \frac{\partial Y_{j}}{\partial t}=D_{j} \Delta Y_{j}+a_{i} Y_{j}\left(1-\frac{Y_{j}}{K_{j}}\right)+\eta_{j} Y_{j} Y_{j-1}-\mu_{j+1} Y_{j} Y_{j+1}, \\
& \frac{\partial Y_{n}}{\partial t}=D_{n} \Delta Y_{n}+a_{n} Y_{n}+\eta_{n} Y_{n} Y_{n-1},
\end{aligned}
$$

After rescaling Equation (3) it can be written in the following simplified form

$$
\begin{aligned}
\frac{\partial y_{0}}{\partial t}(t, x)= & y_{0}(t, x)\left(a\left(1-y_{0}(t, x)\right)-\mu y_{1}(t, x)\right) \\
& +d_{0} \Delta y_{0}(t, x), \\
\frac{\partial y_{1}}{\partial t}(t, x)= & -y_{1}(t, x)+\eta y_{0}(t-\tau, x) y_{1} \\
& \times(t-\tau, x)+d_{1} \Delta y_{1}(t, x),
\end{aligned}
$$

where $y_{0}$ represents the density of normal cells at time $t$, and position $x, y_{1}$ stands for malignant cells, constant

$$
a=\frac{a_{0}}{a_{1}}, \mu=\frac{\mu_{1}}{a}, \eta=\frac{\eta_{1}}{a_{1}}, d_{0}=\frac{d_{0}}{d_{1}}, d_{1}=\frac{D_{1}}{a_{1}}
$$

are positive, delay $\tau=\frac{\tau_{s}}{a_{1}}$ is non-negative and $x \in(0, \pi)$, with the Neumann boundary and initial conditions

$$
\begin{aligned}
& \left.\frac{\partial y_{i}}{\partial t}(t, x)\right|_{x=0, \pi}=0, i=0,1 \\
& y_{i}(t, x)=\varphi_{i}(t, x)>0, t \in[-\tau, 0], x \in[0, \pi], i=0,1 .
\end{aligned}
$$

It is reasonable to consider interaction not only between cells on subsequent stages of mutations, and therefore we consider the following two-stage model

$$
\begin{aligned}
\frac{\partial y_{0}}{\partial t}(t, x)= & y_{0}(t, x)\left(a_{0}\left(1-y_{0}(t, x)\right)-\mu_{1} y_{1}(t, x)\right. \\
& \left.-\xi_{1} y_{2}(t, x)\right)+d_{0} \Delta y_{0}(t, x), \\
\frac{\partial y_{1}}{\partial t}(t, x)= & y_{1}(t, x)\left(a_{1}\left(1-y_{1}(t, x)\right)-\mu_{2} y_{2}(t, x)\right) \\
& +\eta_{1} y_{0}\left(t-\tau_{1}, x\right) y_{1}(t-\tau, x)+d_{1} \Delta y_{1}(t, x), \\
\frac{\partial y_{2}}{\partial t}(t, x)= & -y_{2}(t, x)+\eta_{2} y_{1}\left(t-\tau_{2}, x\right) y_{1}\left(t-\tau_{2}, x\right) \\
& -\xi_{2} y_{0}(t, x) y_{2}(t, x)+d_{2} \Delta y_{2}(t, x),
\end{aligned}
$$

subject to

$$
\begin{aligned}
& \left.\frac{\partial y_{i}}{\partial t}(t, x)\right|_{x=0, \pi}=0, \quad i=0,1,2 \\
& y_{i}(t, x)=\varphi_{i}(t, x)>0, t \in[-\tau, 0], x \in[0, \pi], i=0,1,2 .
\end{aligned}
$$

\section{Spectral Postprocessing Technique}

In this section, we will describe in detail the spectral methods for Equations (4) and (5). We first use a finite difference scheme in time and spectral methods in space.

\subsection{Finite Difference Scheme}

Let $x_{j}=\frac{\pi\left(1+s_{j}\right)}{2}, t_{n}=n \Delta t$ with $\left\{s_{j}\right\}_{j=0}^{M}$ are are Legendre-Gauss-Lobatto points in $[-1,1]$ and $\Delta t=\frac{T}{N}$. Without losing any generality, we can choose $N$ such that $\tau=k \Delta t$, where $k$ is an integer. Denote by

$$
\begin{aligned}
& y_{1, j+1}^{n+1} \approx y_{1}\left(t_{n+1}, x_{j+1}\right), y_{0, j}^{n} \approx y_{1}\left(t_{n}, x_{j}\right), \\
& y_{1}^{n}=\left[y_{1,0}^{n}, \cdots, y_{1, M}^{n}\right]^{\mathrm{T}}, y_{0}^{n}=\left[y_{0,0}^{n}, \cdots, y_{0, M}^{n}\right]^{\mathrm{T}},
\end{aligned}
$$


then the implicit difference scheme is given by enforcing Equation (4) at $\left(t_{n+1}, x_{j}\right)$

$$
\begin{aligned}
& \frac{y_{1, j}^{n+1}-y_{1, j}^{n}}{\Delta t}=-y_{1, j}^{n+1}+d_{1} D_{2} y_{1}^{n+1} \\
& +\eta y_{0, j}^{n+1-k} y_{1, j}^{n+1-k} \quad \text { for } 1 \leq j \leq M-1,
\end{aligned}
$$

where $D_{2}=\left(\frac{2}{\pi} D\right)^{2}$ with $D$ is the differential ma trix associated with Legendre-Gauss-Lobatto nodes, see [13] for details. Rearranging Equation (6) in terms of matrix form, we have

$$
\left((1+\Delta t) I-d_{1} D_{2}\right) y_{1}^{n+1}=y_{1}^{n}+\Delta t_{n} \eta y_{0}^{n+1-k} \odot y_{1}^{n+1-k}
$$

where $\odot$ means element-wise multiplication of two vectors. Here we should remark, considering the boundary conditions in Equation (4), that is the first and the last equation in the above equation, that is Equation (7) do not hold any more. Instead, we must replace those two equations by directly discretizing the boundary conditions respectively. Similarly the semi-implicit scheme for $y_{0, j}^{n+1}$ is given by

$$
\left(I-\Delta t d_{1} D_{2}\right) y_{0}^{n+1}=y_{0}^{n}+\Delta t y_{0}^{n} \odot\left(a\left(1-y_{0}^{n}\right)-\mu y_{1}^{n+1}\right),
$$

where we utilize the updated $y_{1}^{n+1}$ in Equation (7). Moreover, to replace the first and the last equations in Equation (8), we also need to enforce the boundary conditions of $y_{0}$ that

$$
D_{0},: y_{1}^{n+1}=0 \text { and } D_{M},: y_{1}^{n+1}=0 .
$$

Applying the same implicit and semi-implicit finite difference scheme to the two-stage mutation model, we have the following system

$$
\begin{aligned}
& \left((I+\Delta t) I-\Delta t d_{2} D_{2}\right) y_{2}^{n+1} \\
& =y_{2}^{n}+\Delta t \eta_{2} y_{1}^{n+1-k_{2}} \odot y_{2}^{n+1-k_{2}}-\Delta t \xi_{2} y_{0}^{n} \odot y_{2}^{n}, \\
& \left(I-\Delta t d_{1} D_{2}\right) y_{1}^{n+1} \\
& =y_{1}^{n}+\Delta t y_{1}^{n} \odot\left(a_{1}\left(1-y_{1}^{n}\right)-\mu_{2} y_{2}^{n+1}\right) \\
& +\Delta t \eta_{1} y_{0}^{n+1-k_{1}} \odot y_{1}^{n+1-k_{1}}, \\
& \left(I-\Delta t d_{0} D_{2}\right) y_{0}^{n+1} \\
& =y_{0}^{n}+\Delta t y_{0}^{n} \odot\left(a_{0}\left(1-y_{0}^{n}\right)-\mu_{1} y_{1}^{n+1}-\xi_{1} y_{2}^{n+1}\right)
\end{aligned}
$$

where $k_{i}=\frac{\tau_{i}}{\Delta t}$ are assumed to be integer for simplicity. In fact, we can deal with any $\tau_{i}$ by interpolation if there is no suitable $\Delta t$ such that $k_{i}$ be integers. Moreover, to satisfy the boundary conditions which is similar to one-stage mutation model, we can replace the first and the last equations in each matrix form by

$$
D_{0},: y_{1}^{n+1}=0 \text { and } D_{M},: y_{1}^{n+1}=0,
$$

for $i=0,1,2$, respectively.

\section{Postprocessing}

It is well known that backward Euler finite difference scheme in time direction is of the first order accuracy, which is much worse than the spectral accuracy in spatial direction. Therefore, to achieve the balance between the errors in two directions, we use spectral postprocessing in [13] to enhance the accuracy in time direction based upon the backward Euler finite difference scheme.

To describe the time marching scheme clearly, we give some notations as follows. Split the time interval $[0, T]$ into several subintervals, i.e., $[0, T]=U_{i}\left[t_{p_{i}}, t_{p_{i+1}}\right]$, for simplicity we can take $\tau=t_{p_{i+1}}-t_{p_{i}}$. Let $\theta_{i j}=t_{p_{i}}+\frac{\tau}{2}\left(1+s_{j}\right)$ with $s_{j}$ given above as LegendreGauss-Lobatto points in $[-1,1]$.

Integrating Equation (4) from $t_{p_{i}}$ to $\theta_{i k}$, we get

$$
\begin{aligned}
& \int_{t_{p_{i}}}^{\theta_{i k}} \frac{\partial}{\partial t} y_{1}(\theta, x) \mathrm{d} \theta=-\int_{t_{p_{i}}}^{\theta_{i k}} y_{1}(\theta, x) \mathrm{d} \theta \\
& +\int_{t_{p_{i}}}^{\theta_{i k}} \eta y_{0}\left(\theta-\tau, x_{j}\right) y_{1}\left(\theta-\tau, x_{j}\right) \mathrm{d} \theta \\
& +d_{1} \int_{t_{p_{i}}}^{\theta_{i k}} \frac{\partial^{2}}{\partial x^{2}} y_{1}(\theta, x) \mathrm{d} \theta
\end{aligned}
$$

using the $(N+1)$-point Legendre Gauss-Lobatto quadrature formula relative to the Legendre weights on the right hand side of Equation (10) gives

$$
\begin{aligned}
& y_{1}\left(\theta_{i k}, x_{j}\right)=y_{1}\left(t_{p_{i}}, x_{j}\right)+\frac{\tau}{4}\left(1+s_{k}\right) \\
& \times \sum_{m=0}^{M} \eta y_{0}\left(\rho_{i k m}-\tau, x_{j}\right) y_{1}\left(\rho_{i k m}-\tau, x_{j}\right) \\
& -y_{1}\left(\rho_{i k m}, x_{j}\right)+d_{1} \sum_{n=0}^{M} D 2_{j k} y_{1}\left(\rho_{i k m}, x_{k}\right) \omega_{m},
\end{aligned}
$$

where $\rho_{i k m}=t_{p_{i}}+\frac{\tau}{4}\left(1+s_{k}\right)\left(1+s_{m}\right)$. In the above formula, to explicitly update $y_{1}\left(\theta_{i k}, x_{j}\right)$, we need to evaluate $y_{1}\left(\rho_{i k m}, x_{j}\right)$ by some approximations. Fortunately, we have the Euler scheme solution (7) as an initial approximation and then interpolation to the LegendreGauss-Lobatto points in $\left[t_{p_{i}}, t_{p_{i+1}}\right]$. Denote by $y_{1}^{n}\left(\theta_{i k}, x_{j}\right)$ the $n$-th loop of post-processing solution, whereas for $n=0$, it is the linear interpolation of the solution (7). The full numerical scheme for Equation (4) 
is given by

$$
\begin{aligned}
& y_{1}\left(\theta_{i k}, x_{j}\right)=y_{1}^{n}\left(t_{p_{i}}, x_{j}\right)+\frac{\tau}{4}\left(1+s_{k}\right) \\
& \times \sum_{m=0}^{M}\left(\eta \sum_{e=0}^{M} y_{1}^{N}\left(\theta_{i-1, e}, x_{j}\right) F_{e}\left(\rho_{i k m}-\tau\right)\right)^{2} \\
& \times y_{1}^{N}\left(\theta_{i-1, e}, x_{j}\right)-\left(\sum_{e=0}^{k-1} y_{1}^{n}\left(\theta_{i-1, e}, x_{j}\right) F_{e}\left(\rho_{i k m}\right)\right. \\
& \left.+\sum_{e=k}^{M} y_{1}^{n-1}\left(\theta_{i e}, x_{j}\right) F_{e}\left(\rho_{i k m}\right)\right) \\
& +d_{1} \sum_{k=0}^{M} D 2_{j k}\left(\sum_{e=0}^{k-1} y_{1}^{n}\left(\theta_{i e}, x_{k}\right) F_{e}\left(\rho_{i k m}\right)\right. \\
& \left.+\sum_{e=k}^{M} y_{1}^{n-1}\left(\theta_{i e}, x_{j}\right) F_{e}\left(\rho_{i k m}\right)\right) \omega_{m},
\end{aligned}
$$

where $F_{e}(\cdot)$ is the $e$-th Lagrangian interpolation polynomials associated with Legendre-Gauss-Lobatto points. Here we remark that in Equation (12) the updated information of $y_{1}^{n}\left(\theta_{i k}, x_{j}\right)$ is immediately used to obtain $y_{1}\left(\theta_{i, k+1}, x_{j}\right)$. Similarly, we can get the spectral post processing scheme for $y_{0}$ in Equation (4):

$$
\begin{aligned}
& y_{0}^{n}\left(\theta_{i k}, x_{j}\right)=y_{0}^{n}\left(t_{p_{i}}, x_{j}\right)+\frac{\tau}{4}\left(1+s_{k}\right) \\
& \times \sum_{m=0}^{M}\left(y_{0, t e m p, j} a\left(1-y_{0, t e m p, j}\right)-\mu y_{0, t e m p, j}\right) \\
& +d_{0} \sum_{n=0}^{M} D 2_{j k} y_{1}\left(\rho_{i k m}, x_{k}\right) \omega_{m}
\end{aligned}
$$

where

$$
\begin{aligned}
& y_{0, \text { temp }, j}=\sum_{e=0}^{k-1} y_{0}^{n}\left(\theta_{i e}, x_{j}\right) F_{e}\left(\rho_{i k m}\right) \\
& +\sum_{e=k}^{M} y_{0}{ }^{n-1}\left(\theta_{i e}, x_{j}\right) F_{e}\left(\rho_{i k m}\right) \\
& \text { and } \\
& y_{1, t e m p, j}=\sum_{e=0}^{k} y_{1}^{n}\left(\theta_{i e}, x_{j}\right) F_{e}\left(\rho_{i k m}\right) \\
& +\sum_{e=k+1}^{M} y_{1}^{n-1}\left(\theta_{i e}, x_{j}\right) F_{e}\left(\rho_{i k m}\right)
\end{aligned}
$$

For the sake of compactness, we shall not give the full spectral post processing scheme for the two stage Equation (5) here. However, the idea is same as the one stage model. Instead, we give an algorithm to implement the spectral post processing scheme in detail.

\section{Algorithm 5.1 (Spectral Post-Processing)}

1) Initialize $y_{1}^{0}\left(\theta_{0,0}, x_{j}\right)$ and $y_{0}^{0}\left(\theta_{0,0}, x_{j}\right)$ by interpo- lation from Equations (7) and (8)

2) For $i=1$ to $\#$ of subintervals

3) For $n=0$ to \# of loops on post processing

4) For $k=0$ to $M$

5) $\theta_{i k} \leftarrow t_{p_{i}}+\frac{\tau}{2}\left(1+s_{k}\right), \rho_{i k m} \leftarrow t_{p_{i}}+\frac{\tau}{4}\left(1+s_{k}\right)\left(1+s_{m}\right)$

6) Compute $y_{1}\left(\theta_{i k}, x_{j}\right)$ by Equation (12)

7) Compute $y_{0}\left(\theta_{i k}, x_{j}\right)$ by Equation (13)

8) End $k$

9) End $n$

10) End $i$

\section{Numerical Simulation and Discussion}

In the following, some numerical simulations were carried out. In our computations we use the Legendre-Gauss quadrature with weights

$$
\omega_{j}=\frac{1}{\left(1-x_{j}^{2}\right)\left[L_{N+1}\left(x_{j}\right)\right]^{2}}, 0 \leq j \leq N .
$$

In case of one-stage model the initial functions $\phi_{0}(t, x)=0.2+0.1 \cos (4 \pi x), \phi_{1}(t, x)=1.77$ for healthy and cancer cells respectively and the parameters value

$$
a=2, d_{0}=1 \mathrm{e}^{-6}, d_{1}=4 \mathrm{e}^{-6}, \mu=0.9, \eta=5 .
$$

and for the two-stage model, we use

$$
\begin{aligned}
& a_{0}=2, a_{1}=1, d_{0}=1 \mathrm{e}^{-6}, d_{1}=4 \mathrm{e}^{-6}, \\
& d_{2}=4 \mathrm{e}^{-6}, \mu_{1}=0.5, \mu_{2}=0.25, \eta_{1}=2, \\
& \eta_{2}=3, \xi_{1}=0.1, \xi_{2}=0.2 .
\end{aligned}
$$

with

$$
\begin{aligned}
& \phi_{0}(t, x)=0.2+0.1 \cos (4 \pi x), \phi_{1}(t, x)=1.77 \\
& \phi_{2}(t, x)=1.77
\end{aligned}
$$

We start our simulation for the one-stage model, which is system of Equation (4) with small delay in which the steady state is positive; we observe the oscillatory behavior of the system with different mode of frequencies. The oscillation is then smooth because of the steady state (Figures 1 and 2). Increasing the delay term and fixing the other parameters, one can observe a very strong oscillatory behavior of the system. For a very large value of delay term, the solution behaves like traveling waves (Figures 3 and 4). As the dynamics of the system is period, it suggests that traveling wave solutions appear for the system with delay and diffusion. The same was observed for the two-stage model, which is system Equation (5) (Figures 5-10). In conclusion, we can say that the stability switch is only because of the increasing in delay and the diffusion itself cannot destabilize the system. 


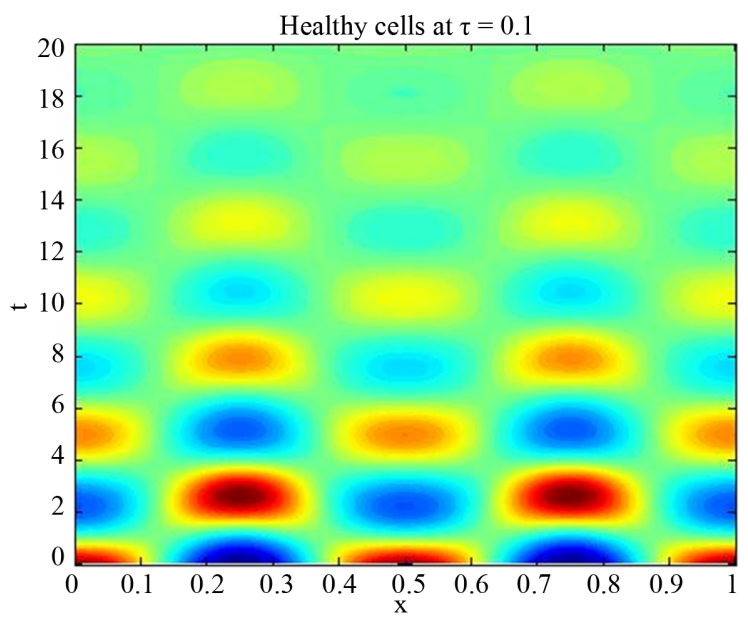

Figure 1. Simulated solution of system (4) for $\tau=0.1$, using the parameters values of Equation (14).

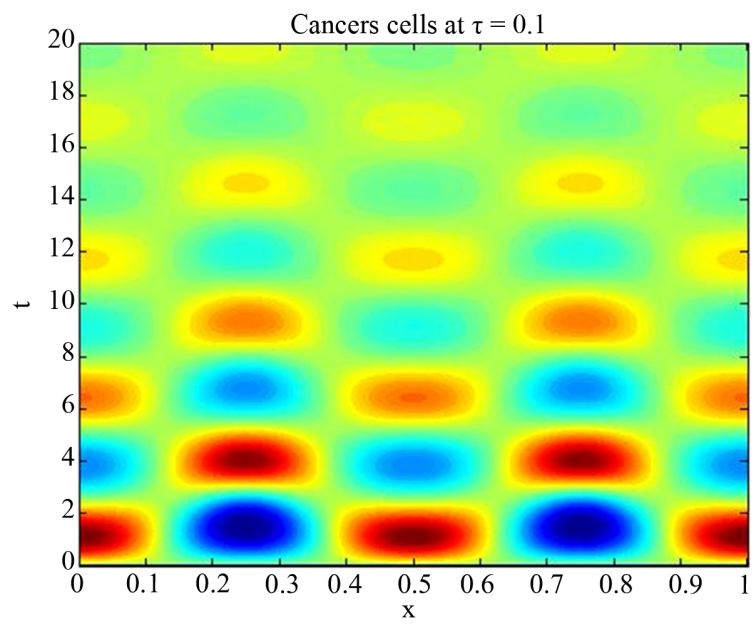

Figure 2. Simulated solution of system (4) for $\tau=0.1$, using the parameters values of Equation (14).

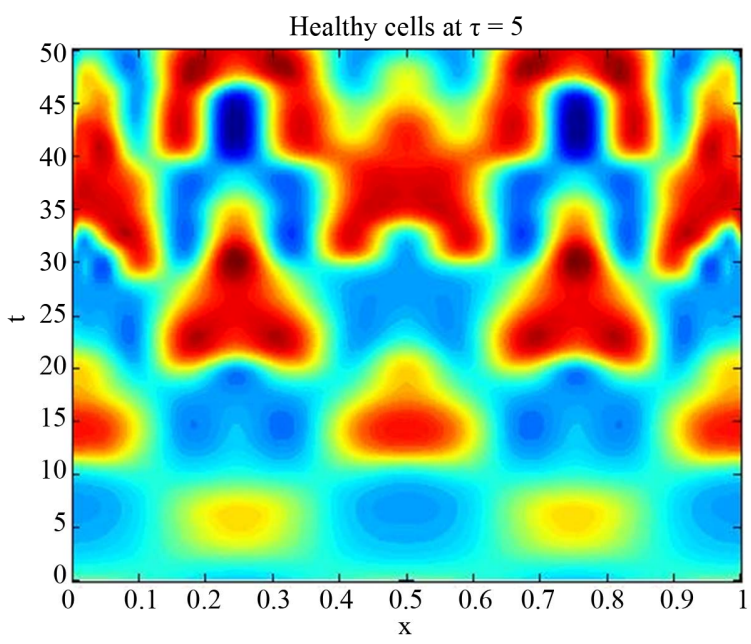

Figure 3. Simulated solution of system (4) for $\tau=5$, using the parameters values of Equation (14).

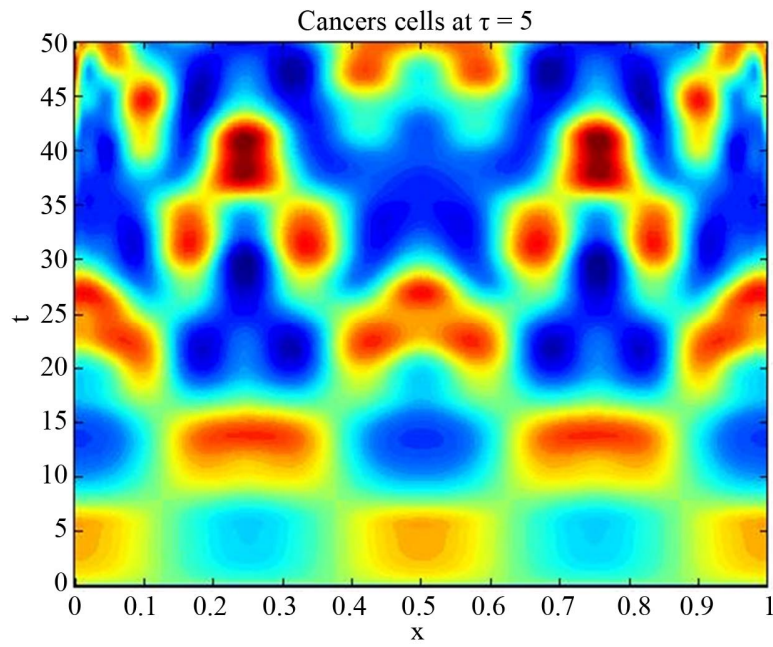

Figure 4. Simulated solution of system (4) for $\tau=5$, using the parameters values of Equation (14).

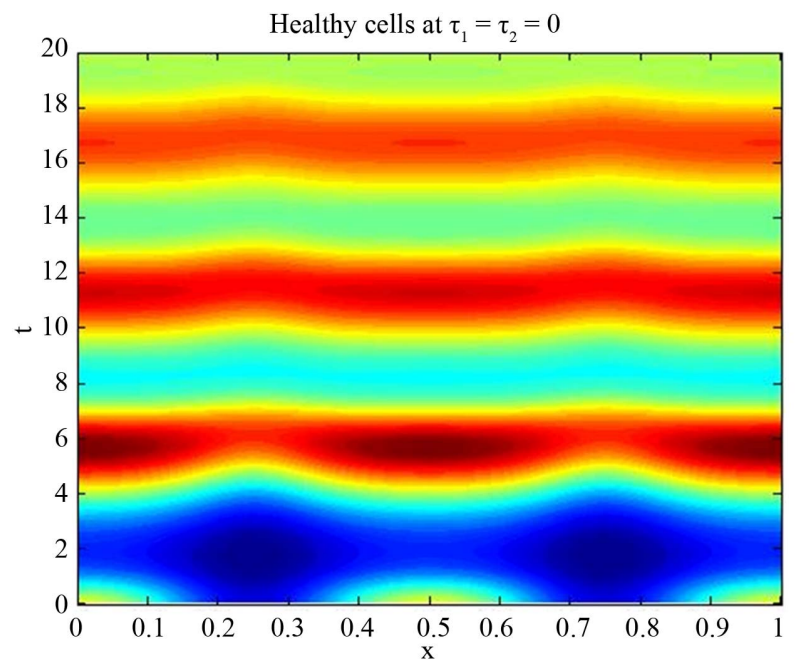

Figure 5. Simulated solution of system (5) for $\tau_{1}=\tau_{2}=0$, using the parameters values of Equation (15).

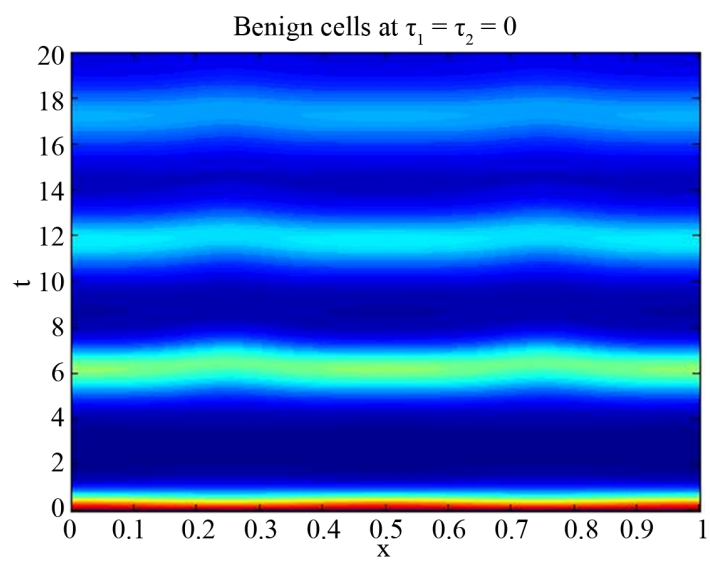

Figure 6. Simulated solution of system (5) for $\tau_{1}=\tau_{2}=0$, using the parameters values of Equation (15). 


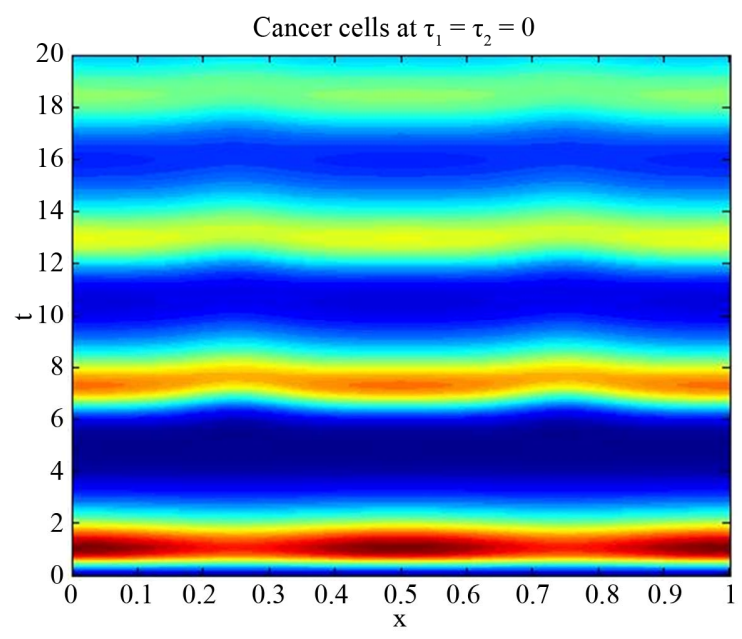

Figure 7. Simulated solution of system (5) for $\tau_{1}=\tau_{2}=0$, using the parameters values of Equation (15).

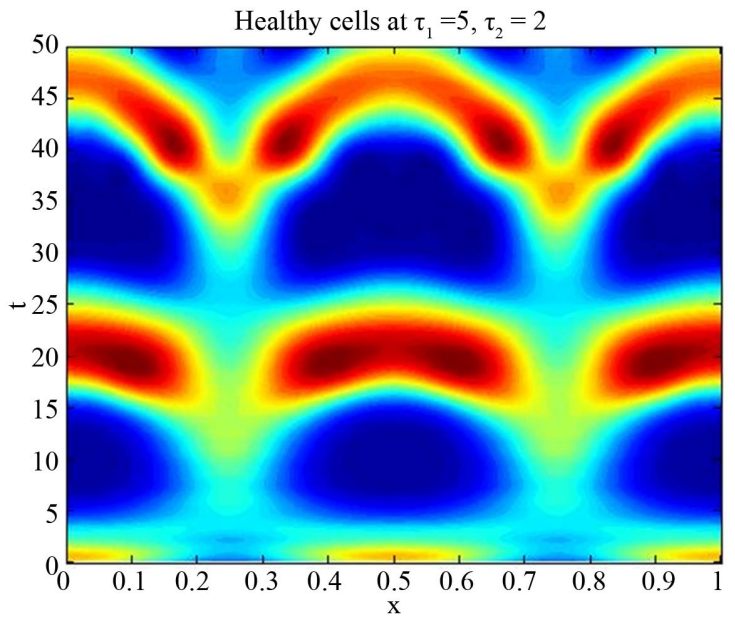

Figure 8. Simulated solution of system (5) for $\tau_{1}=5, \tau_{2}=2$, using the parameters value of Equation (15).

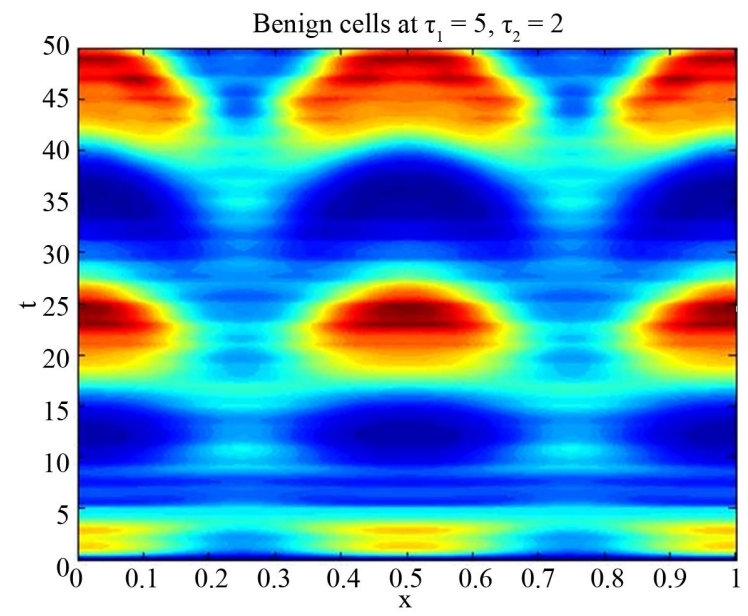

Figure 9. Simulated solution of system (5) for $\tau_{1}=5, \tau_{2}=2$, using the parameters value of Equation (15).

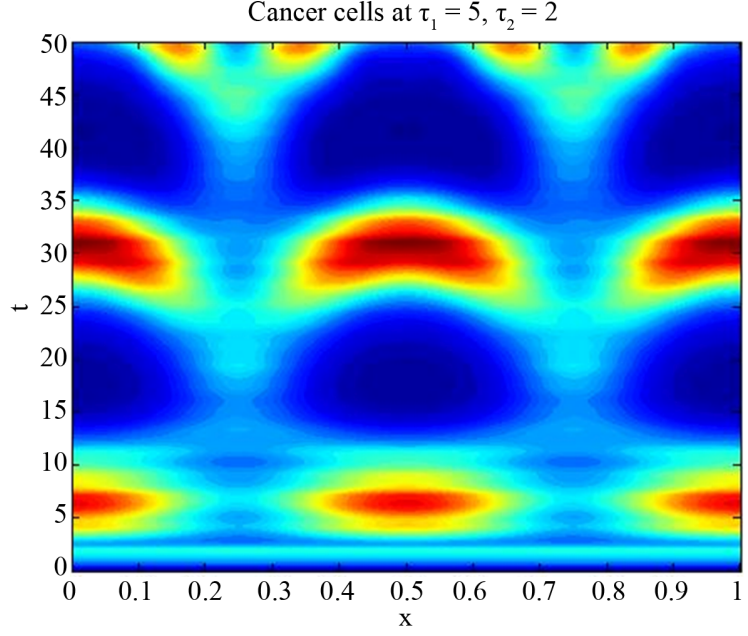

Figure 10. Simulated solution of system (5) for $\tau_{1}=5$, $\tau_{2}=2$ using the parameters value of Equation (15).

\section{Concluding Remarks}

It has been the aim of this paper to use a spectral postprocessing technique for the numerical solutions of one and two-stage model of carcinogenesis mutations with time delay and diffusion. The spectral postprocessing with the coarse-mesh symplectic initial guess produces high accurate approximate solution. It also saves a significant amount of computational time over the standard schemes. We compared the results obtained by simulateing the one and two-stage model with the available one and find it with good agreement. The future work includes the theoretical stability analysis of the proposed method and its extension to higher dimensions.

\section{Acknowledgements}

The author is very grateful to Professor Urszula Forys of the Institute of Applied Mathematics and Mechanics Faculty of Mathematics, Informatics and Mechanics University of Warsaw, Poland for her many discussions and $\mathrm{Xu}$ Xiang of MSU for his help in Algorithm.

\section{REFERENCES}

[1] J. C. Arciero, T. L. Jackson and D. E. Kirschner, "A Mathematical Model of Tumor-Immune Evasion and siRNA Treatment," Discrete and Continuous Dynamical Systems, Series B, Vol. 4, No. 1, 2004, pp. 39-58.

[2] S. Kruś, "Pathological Anathomy," PZWL, Warsaw, 2001.

[3] R. Ahangar and X. B. Lin, "Multistage Evolutionary Model for Carcinogenesis Mutations," Electronic Journal of Differential Equations, Conference 10, 2003, pp. 33-53.

[4] M. Bodnar, U. Foryś, M. J. Piotrowska and J. Poleszczuk, "A Simple Model of Carcinogenesis Mutations with Time Delay and Diffusion," Mathematical Biosciences and Engineering, Vol. 10, No. 3, 2013, pp. 861-872. 
http://dx.doi.org/10.3934/mbe.2013.10.861

[5] U. Foryś, "Biological Delay Systems and the Mikhailov Criterion of Stability," Journal of Biological Systems, Vol. 12, No. 1, 2004, pp. 45-60. http://dx.doi.org/10.1142/S0218339004001014

[6] U. Foryś, "Stability Analysis and Comparison of the Models for Carcinogenesis Mutations in the Case of TwoStages of Mutations," Journal of Applied Analysis, Vol. 11, No. 2, 2005, pp. 200-281. http://dx.doi.org/10.1515/JAA.2005.283

[7] U. Foryś, "Multi-Dimensional Lotka-Volterra System for Carcinogenesis Mutations," Mathematical Methods in the Applied Sciences, Vol. 32, No. 17, 2009, pp. 2287-2308. http://dx.doi.org/10.1002/mma.1137

[8] U. Foryś, "Comparison of the Models for Carcinogenesis Mutations-One-Stage Case," The Proceeding of the 5th National Conference on Mathematics, Applied to Biology and Medicine, Święty Krzyż, 2004.
[9] J. D. Murry, "Mathematical Biology 1: An Introduction," Springer, Berlin, 2002.

[10] J. D. Murry, "Mathematical Biology 11: Special Models and Biochemical Applications, Interdisciplinary Applied Mathematics," Springer, Berlin, 2003.

[11] J. D. Murry, "Mathematical Biology 11: Special Models and Biochemical Applications, Interdisciplinary Applied Mathematics," Springer, Berlin, 2003.

[12] M. R. Garvie, "Finite-Difference Schemes for ReactionDiffusion Equations Modeling Redator-Prey Interactions in MATLAB," Bulletin of Mathematical Biology, Vol. 69 , No. 3, 2007, pp. 931-956.

http://dx.doi.org/10.1007/s11538-006-9062-3

[13] T. Tang and X. Xu, "Accuracy Enhancement Using Spectral Postprocessing for Differential Equations and Integral Equations," Communications in Computational Physics, Vol. 5, 2009, pp. 779-792. 